\title{
INTRODUCTION AUX PRODUITS TENSORIELS DE FONCTIONNELLES CONVEXES
}

\author{
MarC ATTEIA ${ }^{1}$
}

\begin{abstract}
This paper gives new results on tensor products of convex functionals. It extends the work of Alexandre Grothendieck regarding to tensor products of semi-norms contained in his thesis on tensor products of topological spaces [5] and in his paper about the metric theory of topological tensor products [6].

This paper provides basic tools for the study of biconvex functionals which play an essential role in many parts of experimental sciences.

Résumé. Cet article présente des résultats nouveaux que j'ai obtenus dans l'étude systématique des produits tensoriels de fonctionnelles convexes. Il étend l'étude des produits tensoriels de semi-normes faite par Alexandre Grothendieck dans sa thèse sur les produits tensoriels topologiques [5] et dans son article sur la théorie métrique des produits tensoriels topologiques [6]. Cet article fait référence à des travaux de recherche que j'ai dirigés dans le cadre du Laboratoire d'Analyse Numérique de l'Université Paul Sabatier ( [2-4]). Il pose les bases d'une théorie des fonctionnelles biconvexes qui jouent un rôle fondamental dans différentes branches des sciences expérimentales.
\end{abstract}

\section{Introduction}

1.1 Dans deux notes aux CRAS, publiées en 1973, j'ai présenté les bases de l'analyse convexe projective ; j'y explicitais formellement le fait que la théorie classique des fonctionnelles convexes était, dans un certain sens, une extension de la théorie élémentaire des espaces vectoriels topologiques localement convexes.

Dans une de ces notes, je montrais, en particulier, que le théorème de Dieudonné sur la fermeture de la somme de deux ensembles convexes fermés, dans un espace vectoriel topologique localement convexe, se déduisait par dualité projective du théorème fondamental de Ky-Fan.

(La théorie des fonctionnelles convexes apparaît donc, ainsi, comme une extension de la théorie classique des semi-normes, elle-même extension de la théorie des fonctionnelles quadratiques).

1.2 Il était donc naturel de développer une théorie des produits tensoriels de fonctionnelles convexes en s'inspirant de la théorie des produits tensoriels de semi-normes dans la thèse d'A. Grothendieck.

Dans cette perspective, j'ai dirigé plusieurs travaux, dans le cadre du Laboratoire d'Analyse numérique de Toulouse. Je citerai principalement :

(1) La thèse de J. P. Dedieu [3], qui établissait les premières propriétés essentielles des produits tensoriels $\pi$ et $\varepsilon$ de deux fonctionnelles convexes.

(2) La thèse d'A. El Qortobi [4], qui étudiait en particulier les produits tensoriels $\pi$ et $\varepsilon$ de deux fonctionnelles convexes par tranches.

\footnotetext{
1 1, Rue Clément Ader. 31520 Ramonville-Saint-Agne France; e-mail: marc.atteia@wanadoo.fr
} 
(3) L'article de M. Atteia et M. Raïssouli [2], qui mettait en évidence et étudiait un nouveau produit tensoriel autodual $\sigma$ de deux fonctionnelles convexes.

(4) L'étude des fonctionnelles tensoriellement convexes et ses applications à l'élasticité non linéaire et au contrôle, récemment publiée, [1].

Dans l'article qui suit, nous présentons les propriétés des produits tensoriels $\pi, \varepsilon$ et $\sigma$ de fonctionnelles convexes; nous montrons aussi comment les idées développées par A. Grothendieck dans son "Résumé de la théorie métrique des produits tensoriels topologiques" peuvent être étendues au cas des produits tensoriels de fonctionnelles convexes. Nous en déduisons en particulier, dans quelles conditions on a : $\pi \leq \sigma \leq \varepsilon$.

1.3 On sait qu'étant donné trois espaces vectoriels $E, F, G$ et une application bilinéaire $B$ de $E \times F$ dans $G$, il existe une application linéaire $L$ de $E \otimes F$ dans $G$ telle que :

$$
\forall(x, y) \in E \times F, B(x, y)=L(x \otimes y) .
$$

Etendre cette propriété au cas des fonctionnelles biconvexes semble naturel.

Notons que si $f$ (resp. $g$ ) est une fonctionnelle convexe sur $E$ (resp. $F$ ), l'application qui de $E \times F$ dans $\mathbb{R}$ qui à tout $(x, y) \in E \times F$ associe $f(x) . g(y)$ est biconvexe.

Avec des hypothèses convenables, si $\gamma=\pi, \sigma, \varepsilon$, on a :

$$
\forall(x, y) \in E \times F,(f \gamma g)(x \otimes y)=f(x) \cdot g(y)
$$

Si $Q$ est une fonctionnelle biconvexe sur $E \times F$, en général il n'existe donc pas de fonctionnelle convexe unique $\Phi$ sur $E \otimes F$ telle que :

$$
\forall(x, y) \in E \times F, Q(x, y)=\Phi(x \otimes y) .
$$

Nous expliciterons dans un article ultérieur les liens (que nous avons établis) entre les fonctionnelles biconvexes (multiconvexes) et les produits tensoriels ainsi que les applications à la mécanique ou à la physique. On trouvera un type de résultat, dans ce sens, dans l'article cité en [1].

\section{RAPPELS SUR LES PRODUITS TENSORIELS DE SEMI-NORMES.}

Définition 2.1. Soient $(E, p)$ et $(F, q)$ deux espaces vectoriels semi-normés. On pose:

$$
\forall z \in E \otimes F, \quad(p \pi q)(z)=\operatorname{Inf}\left\{\sum_{j} p\left(x_{j}\right) \cdot q\left(y_{j}\right) ; z=\sum_{j}\left(x_{j} \otimes y_{j}\right)\right\} .
$$

D'où, en particulier : $\forall(x, y) \in E \times F,(p \pi q)(x \otimes y)=p(x) \cdot q(y)$.

$\pi$ est appelée la semi-norme projective sur le produit tensoriel de $(E, p)$ et $(F, q)$.

$(E, p)$ étant un espace semi-normé, on pose : $S(p ; 1)=\{x \in E ; p(x) \leq 1\}$.

On montre que :

Proposition 2.1. $S(p \pi q ; 1)=c o(S(p ; 1) \otimes S(q ; 1))$.

Soit $E^{*}\left(\right.$ resp. $\left.F^{*}\right)$ le dual topologique de $(E, p)(\operatorname{resp} .(F, q))$ et $<., .>_{E}\left(\right.$ resp. $\left.<. . .>_{F}\right)$ le crochet de dualité entre $(E, p)$ et $E^{*}\left(r e s p .(F, q)\right.$ et $\left.F^{*}\right)$.

On désigne $\operatorname{par} p^{o}\left(\right.$ resp. $\left.q^{o}\right)$, la semi-norme duale de $p($ resp. $q)$ sur $E^{*}\left(\right.$ resp. $\left.F^{*}\right)$.

On pose : $\forall(x, y) \in E \times F, \forall\left(x^{*}, y^{*}\right) \in E^{*} \times F^{*}$,

$$
<x \otimes y, x^{*} \otimes y^{*}>=<x, x^{*}>_{E} \cdot<y, y^{*}>_{F}
$$




$$
\begin{aligned}
& \text { et } \forall z=\sum_{j}\left(x_{j} \otimes y_{j}\right) \in E \otimes F, \forall z^{*}=\sum_{k}\left(x_{k}^{*} \otimes y_{k}^{*}\right) \in E^{*} \otimes F^{*}, \\
&<z, z^{*}>_{\otimes}=\sum_{j, k}<x_{j} \otimes y_{j}, x_{k}^{*} \otimes y_{k}^{*}>_{\otimes} .
\end{aligned}
$$

Alors,

Définition 2.2. On pose $\forall z \in E \otimes F$, $(p \varepsilon q)(z)=\operatorname{Sup}\left\{<z, x^{*} \otimes y^{*}>\otimes ;\left(x^{*}, y^{*}\right) \in E^{*} \times F^{*}, p^{o}\left(x^{*}\right) \leq 1, q^{o}\left(y^{*}\right) \leq 1\right\}$ D'où, en particulier :

$\forall(x, y) \in E \times F,(p \varepsilon q)(x \otimes y)=p(x) \cdot q(y)$.

$\varepsilon$ est appelée la semi-norme inductive sur le produit tensoriel de $(E, p)$ et $(F, q)$.

On montre que :

Proposition 2.2. $S(p \varepsilon q ; 1)=\left((S(p ; 1))_{E}^{o} \otimes(S(q ; 1))_{F}^{o}\right)_{\otimes}^{o}$ où : $(.)_{E}^{o}\left(\right.$ resp. $\left.\left.(.)_{F}^{o}\right),(.)_{\otimes}^{o}\right)$ désigne la polarité relative $\grave{a}<., .>_{E}\left(\right.$ resp. $\left.\left.<., .>_{F},\right)<., .>_{\otimes}\right)$.

De même :

Proposition 2.3. Quels que soient les espaces semi-normés $(E, p)$ et $(F, q), \forall z \in E \otimes F$, on a:

$$
(p \varepsilon q)(z) \leq(p \pi q)(z) .
$$

On dira que $\varepsilon \leq \pi$.

Définition 2.3. On dira qu'une semi-norme $\gamma$ sur $E \otimes F$ est raisonnable si $\varepsilon \leq \gamma \leq \pi$, c'est à dire :

$$
\forall z \in E \otimes F,(p \varepsilon q)(z) \leq(p \gamma q)(z) \leq(p \pi q)(z) .
$$

Remarque 2.1. On notera, dans la suite, $E \gamma F$, le produit tensoriel $E \otimes F$ muni de la semi-norme $\gamma$ et on notera $E \widehat{\gamma} F$ un complété de $E \gamma F$.

Soit $\gamma$ telle que $: \varepsilon \leq \gamma \leq \pi$. Alors :

$$
(E \varepsilon F)^{*} \subset(E \gamma F)^{*} \subset(E \pi F)^{*} .
$$

D'autre part, si $z \in E \otimes F$, on a :

$$
\forall\left(x^{*}, y^{*}\right) \in E^{*} \times F^{*},<z, x^{*} \otimes y^{*}>_{\otimes} \leq p^{o}\left(x^{*}\right) \cdot q^{o}\left(y^{*}\right) \cdot(p \varepsilon q)(z) .
$$

Il en résulte que $E \otimes F$ est contenu algébriquement dans $\left(E^{*} \varepsilon F^{*}\right)^{*}$, donc dans $\left(E^{*} \gamma F^{*}\right)^{*}$. Si $\operatorname{dim}(E)<+\infty$ et $\operatorname{dim}(F)<+\infty$, on $a: E \otimes F=\left(E^{*} \gamma F^{*}\right)^{*}$.

\section{Produit TENSORIEL DE DEux CÔNES}

Soit $(E, p)$ (resp. $(F, q))$ un espace vectoriel semi-normé, $A$ (resp.B) un cône contenu dans $E$ (resp. $F$ ) ayant l'origine pour sommet.

Définition 3.1. On pose : $A \otimes B=\{z \in E \otimes F ; z=a \otimes b, a \in A, b \in B\}$ et $A \pi B=c o(A \otimes B)$.

Soit $E^{*}\left(\right.$ resp. $\left.F^{*}\right)$ le dual topologique de $(E, p)(\operatorname{resp} .(F, q))$ et

$$
\begin{gathered}
A^{\circ}=\left\{a^{*} \in E^{*} ; \forall a \in A,<a, a^{*}>_{E} \geq 0\right\}, \\
B^{\circ}=\left\{b^{*} \in E^{*} ; \forall b \in B,<b, b^{*}>_{F} \geq 0\right\} .
\end{gathered}
$$


Posons :

$$
\left(A^{\circ} \otimes B^{\circ}\right)^{\circ}=\left\{z \in E \otimes F ; \forall\left(a^{*}, b^{*}\right) \in\left(A^{\circ} \otimes B^{\circ}\right),<z, a^{*} \otimes b^{*}>_{\otimes} \geq 0\right\}
$$

On montre facilement que :

$$
A \otimes B \subset\left(A^{\circ} \otimes B^{\circ}\right)^{\circ} \otimes
$$

Définition 3.2. $A \varepsilon B=\left(A^{\circ} \otimes B^{\circ}\right)^{\circ} \otimes$.

On en déduit que :

$$
\overline{A \pi B}^{\otimes} \subset A \varepsilon B .
$$

Plus généralement, si $\varepsilon \leq \gamma \leq \pi$, posons :

$$
\left(A^{\circ} \otimes B^{\circ}\right)_{\gamma}^{\circ}=\left\{u \in\left(E^{*} \gamma F^{*}\right)^{*} ; \forall\left(a^{*}, b^{*}\right) \in\left(A^{\circ} \otimes B^{\circ}\right), u\left(a^{*} \otimes b^{*}\right) \geq 0\right\}
$$

Alors :

$$
\left(A^{\circ} \otimes B^{\circ}\right)^{\circ}{ }_{\varepsilon} \subset\left(A^{\circ} \otimes B^{\circ}\right)^{\circ}{ }_{\gamma} \subset\left(A^{\circ} \otimes B^{\circ}\right)^{\circ} \pi \text {. }
$$

( Il n'y a pas, a priori, de relation entre $A \otimes B($ ou $A \pi B)$ et $\left(A^{\circ} \otimes B^{\circ}\right)^{\circ} \gamma$ ).

\section{Produits tensoriels De Deux fonctionnelles}

Remarque 4.1. Soit $G$ un espace vectoriel et $Q$ une partie de $G$. On pose : $C_{+}(Q)=\cup_{\lambda \in \mathbb{R}_{+}^{*}} \lambda(Q \times\{1\})$. $C_{+}(Q)$ est un cône épointé contenu dans $G \times \mathbb{R}_{+}^{*}$. Si $\varphi$ est une fonctionnelle sur $G$, on pose :

$$
\begin{gathered}
\operatorname{epi}(\varphi)=\{(x, \varrho) \in G \times \mathbb{R} ; \varphi(x)<\varrho\}, \\
\widehat{\operatorname{epi}}(\varphi)=\{(x, \varrho) \in G \times \mathbb{R} ; \varphi(x) \leq \varrho\}, \\
C_{+}(\varphi)=C_{+}(\operatorname{epi}(\varphi)), \widehat{C_{+}}(\varphi)=C_{+}(\widehat{\operatorname{epi}}(\varphi)) .
\end{gathered}
$$

$C_{+}(\varphi)$ (resp. $\widehat{C_{+}}(\varphi)$ ) est un cône épointé contenu dans $G \times \mathbb{R} \times \mathbb{R}_{+}^{*}$. Soit $G^{*}$ un espace vectoriel en dualité avec $G$ et $\left\langle.,>_{G}\right.$ le crochet de dualité entre $G$ et $G^{*}$.

Posons :

$$
\begin{aligned}
\forall(x, \varrho, \sigma) & \in G \times \mathbb{R} \times \mathbb{R}, \forall\left(x^{*}, \varrho^{*}, \sigma^{*}\right) \in G^{*} \times \mathbb{R} \times \mathbb{R}, \\
& \ll(x, \varrho, \sigma),\left(x^{*}, \varrho^{*}, \sigma^{*}\right) \gg=<x, x^{*}>_{G}+\rho . \rho^{*}+\sigma \cdot \sigma^{*} .
\end{aligned}
$$

Alors, $\ll ., . \gg$ met en dualité $G \times \mathbb{R} \times \mathbb{R}$ et $G^{*} \times \mathbb{R} \times \mathbb{R}$. On posera alors :

$$
\left(C_{+}(\varphi)\right)^{\circ}=\left\{\left(x^{*}, \varrho^{*}, \sigma^{*}\right) \in G^{*} \times \mathbb{R} \times \mathbb{R} ; \quad \forall(x, \varrho, \sigma) \in C_{+}(\varphi),<x, x^{*}>_{G}+\rho . \rho^{*}+\sigma . \sigma^{*} \geq 0\right\} .
$$

(on explicitera, plus loin, le lien qui existe entre $\left(C_{+}(\varphi)\right)^{\circ}$ et $C_{+}\left(\varphi^{*}\right)$ ).

Soit $E$ (resp. $F$ ) un espace vectoriel et $f \in \overline{\mathbb{R}}_{+}^{E}$ (resp. $g \in \overline{\mathbb{R}}_{+}^{F}$ ). Par analogie avec ce qui précède définissons $f \pi g$ comme l'élément de $\overline{\mathbb{R}}_{+}^{E \otimes F}$ tel que : $C_{+}(f \pi g)=C_{+}(f) \pi C_{+}(g)$. Alors

Proposition 4.1. $\forall z \in E \otimes F$,

$(f \pi g)(z)=\operatorname{Inf}\left\{\sum_{j} \alpha_{j} f\left(x_{j}\right) g\left(y_{j}\right) ; z=\sum_{j} \alpha_{j}\left(x_{j} \otimes y_{j}\right)\right.$ ò̀ $\left.\alpha_{j} \geq 0, \sum_{j} \alpha_{j}=1,\left(x_{j}, y_{j}\right) \in E \times F\right\}$.

$f \pi g$ est une fonctionnelle convexe, positive sur $E \otimes F$.

Soit $(E, p)$ (resp. $(F, q))$ un espace vectoriel semi-normé et $E^{*}\left(\right.$ resp. $\left.F^{*}\right)$ son dual topologique. On définit, comme ci-dessus, $<.,>_{E},<.,>_{F},<., .>_{\otimes}$. Soit $f \varepsilon g$ l'élément de $\overline{\mathbb{R}}^{E \otimes F}$ tel que :

$$
\left.C_{+}(f \varepsilon g)=\left(C_{+}(f)\right)^{\circ} \otimes\left(C_{+}(g)\right)^{\circ}\right)^{\circ} \otimes
$$

Alors : 
Proposition 4.2. Si $f \in \overline{\mathbb{R}}_{+}^{E}, f^{*} \in \overline{\mathbb{R}}_{+}^{E^{*}}, g \in \overline{\mathbb{R}}_{+}^{F}, g^{*} \in \overline{\mathbb{R}}_{+}^{F^{*}}$, on $a: \forall z \in E \otimes F$

$$
f \varepsilon g=\operatorname{Sup}\left\{<z, x^{*} \otimes y^{*}>_{\otimes}-f^{\sharp}\left(x^{*}\right) \cdot g^{\sharp}\left(y^{*}\right) ;\left(x^{*}, y^{*}\right) \in E^{*} \times F^{*}\right\}
$$

ò̀ : $f^{\sharp}=f^{*}(-\cdot), g^{\sharp}=g^{*}(-\cdot)$.

On montre que :

Proposition 4.3. $f \varepsilon g=\left(f^{\sharp} \pi g^{\sharp}\right)_{\otimes}^{\sharp}$.

On dira que : $\varepsilon=(\pi)_{\otimes}^{\sharp}$. De l'étude sur les produits tensoriels de cônes, on déduit que :

$$
f \varepsilon g \leq f \pi g .
$$

Définition 4.1. Soit $\gamma$ tel que : $\varepsilon \leq \gamma \leq \pi$. Posons : $f \varepsilon_{\gamma} g=\left(f^{\sharp} \pi g^{\sharp}\right)_{\gamma}^{\sharp}$.

Il n'y a pas, a priori, de relation entre $f \pi g$ et $f \varepsilon_{\gamma} g$.

\section{Premières propriétés AlgÉbriques de $\pi$ ET DE $\varepsilon$}

Soit $(E, p)(\operatorname{resp} .(F, q))$ un espace vectoriel semi-normé et $E^{*}\left(\right.$ resp. $\left.F^{*}\right)$ son dual topologique.

Proposition 5.1. Soit $f, g \in \overline{\mathbb{R}}_{+}^{E}, h \in \overline{\mathbb{R}}_{+}^{F}$ tels que : $f^{\sharp}, g^{\sharp} \in \overline{\mathbb{R}}_{+}^{E^{*}}, h^{\sharp} \in \overline{\mathbb{R}}_{+}^{F^{*}}$.

Si $\lambda \in \mathbb{R}_{+}^{*}$, alors :

$$
\begin{aligned}
(\lambda f) \pi h & =f \pi(\lambda h)=\lambda(f \pi h), \\
(f \cdot \lambda) \pi h & =f \pi(h \cdot \lambda)=(f \pi h) \cdot \lambda .
\end{aligned}
$$

et

$$
\begin{aligned}
(f \cdot \lambda) \varepsilon h & =f \varepsilon(h \cdot \lambda)=(f \varepsilon h) \cdot \lambda \\
(\lambda f) \varepsilon h & =f \varepsilon(\lambda h)=\lambda(f \varepsilon h) .
\end{aligned}
$$

Si $\quad f \leq g$, alors :

$$
f \pi h \leq g \pi h,
$$

et

$$
f \varepsilon h \leq g \varepsilon h .
$$

De plus :

$$
\begin{aligned}
(f+g) \pi h & \geq(f \pi h)+(g \pi h) \\
(f \square g) \varepsilon h & \leq(f \varepsilon h) \square(g \varepsilon h), \\
\text { ò̀ } & : \square=(+)^{\sharp} .
\end{aligned}
$$

6. ITÉRATION DE $\pi$ ET DE $\varepsilon$

Proposition 6.1. Soit $\left(E_{j}, p_{j}\right)_{j \in \mathbb{N}^{*}}$ une famille d'espaces de Banach et $\forall j \in \mathbb{N}^{*}$, $E_{j}^{*}$ le dual topologique de $\left(E_{j}, p_{j}\right)$, et $f_{j} \in \overline{\mathbb{R}}_{+}^{E_{j}}$ telle que $f_{j}^{\sharp} \in \overline{\mathbb{R}}_{+}^{E_{j}^{*}}$. On $a$ :

$$
\text { (i) } \quad\left(f_{1} \pi f_{2}\right) \pi f_{3}=f_{1} \pi\left(f_{2} \pi f_{3}\right) \stackrel{\text { def }}{=} f_{1} \pi f_{2} \pi f_{3},
$$


et

$$
\text { (ii) } \quad\left(f_{1} \varepsilon f_{2}\right) \varepsilon f_{3}=f_{1} \varepsilon\left(f_{2} \varepsilon f_{3}\right) \stackrel{\text { def }}{=} f_{1} \varepsilon f_{2} \varepsilon f_{3} \text {. }
$$

En posant alors :

$$
\forall m \in \mathbb{N}^{*}, \quad f_{1} \pi f_{2} \pi \ldots \pi f_{m}=\left(\left(f_{1} \pi f_{2}\right) \pi \ldots\right) \pi f_{m} \text { ò̀ } \quad f_{1} \pi f_{2} \pi \ldots \pi f_{m} \in \overline{\mathbb{R}}_{+}^{E_{1} \otimes \ldots . \otimes E_{m}},
$$

et

$$
\forall m \in \mathbb{N}^{*}, \quad f_{1} \varepsilon f_{2} \varepsilon \ldots \varepsilon f_{m}=\left(\left(f_{1} \varepsilon f_{2}\right) \varepsilon \ldots\right) \varepsilon f_{m}
$$

On vérifie que:

$$
\begin{aligned}
\forall m \in \mathbb{N}^{*}, \quad f_{1} \varepsilon f_{2} \varepsilon \ldots \varepsilon f_{m}= & \left(\left(f_{1}^{\sharp} \pi f_{2}^{\sharp} \pi \ldots \pi f_{m}^{\sharp}\right)\right)^{\sharp} \\
& \text { ò̀ } \quad f_{1} \varepsilon f_{2} \varepsilon \ldots \varepsilon f_{m} \in \overline{\mathbb{R}}_{+}^{E_{1} \otimes \ldots . . E_{m}}
\end{aligned}
$$

Définition 6.1. Soit $(E, p)$ un espace de Banach, $E^{*}$ son dual topologique et, $f \in \overline{\mathbb{R}}_{+}^{E}$ telle que $f^{\sharp} \in \overline{\mathbb{R}}_{+}^{E^{*}}$. On pose, formellement :

$$
\mathcal{E}=\oplus_{j=0}^{\infty} E^{\otimes j}
$$

où $\quad E^{\otimes 0}=\mathbb{R}, E^{\otimes 1}=E \quad$ et $\forall j \in \mathbb{N}^{*}, j \geq 2, E^{\otimes j}=\underbrace{E \otimes \ldots \otimes E}_{j}$

Et, si $\gamma=\pi$ ou $\gamma=\varepsilon, f_{\gamma}^{\otimes j}=\underbrace{f \gamma \ldots \gamma f}_{j}$.

Formellement, on écrira, alors :

$$
\operatorname{Exp}_{\gamma}(f)=\sum_{j=0}^{\infty} \frac{f_{\gamma}^{\otimes j}}{j !}
$$

et

$$
\log _{\gamma}(1-f)=\sum_{j=1}^{\infty} \frac{f_{\gamma}^{\otimes j}}{j}
$$

On considèrera aussi : $\quad \forall x \in E$

$$
\left(\widetilde{\operatorname{Exp}}_{\gamma} f\right)(x)=\sum_{k=0}^{\infty} \frac{f_{\gamma}^{\otimes k}\left(x^{\otimes k}\right)}{k !}
$$

et

$$
\left(\widetilde{\log }_{\gamma}(1-f)\right)(x)=\sum_{k=1}^{\infty} \frac{f_{\gamma}^{\otimes k}\left(x^{\otimes k}\right)}{k}
$$

7. Moyenne DE $\pi$ ET $\varepsilon$

Définition 7.1. Soit $\left(H,\langle\cdot \mid \cdot\rangle_{H}\right)$ et $\left(K,\langle\cdot \mid \cdot\rangle_{K}\right)$ deux espaces de Hilbert. On appelle produit tensoriel de ces deux espaces, l'espace de Hilbert $\left(H \otimes K,\langle\cdot \mid \cdot\rangle_{H \otimes K}\right)$ tel que :

$$
\forall u=\sum_{j}\left(x_{j} \otimes y_{j}\right) \in H \otimes K, \forall v=\sum_{j}\left(x_{j}^{*} \otimes y_{j}^{*}\right) \in H \otimes K, \quad\langle u, v\rangle_{\otimes}=\sum_{j, k}\left\langle x_{j} \mid x_{k}^{*}\right\rangle_{H} \cdot\left\langle y_{j} \mid y_{k}^{*}\right\rangle_{K} \cdot
$$


Posons :

$$
H \sigma K=\left(H \otimes K,\langle\cdot \mid \cdot\rangle_{H \otimes K}\right)
$$

Une question se pose naturellement : Existe-t-il une relation entre les espaces de Banach $H \pi K, H \varepsilon K$ et l'espace de Hilbert $H \sigma K$ ?

R. Schatten (voir [9]) a répondu à cette question en montrant que, dans un certain sens, $\sigma$ est la moyenne de $\pi$ et de $\varepsilon$.

Nous allons généraliser la notion de moyenne qu'il a introduite pour définir le produit tensoriel $f \sigma g$ moyenne des produits tensoriels $f \pi g$ et $f \varepsilon g$.

\section{Algorithme de construction de $f \sigma g$ à partir de $f \pi g$ et $f \varepsilon g$.}

Soit $W$ un espace vectoriel et $\Gamma_{0}(W)$, le cône convexe des fonctionnelles convexes s.c.i. sur $W$.

Notons $\sharp$ la dualité qui à toute $f \in \Gamma_{0}(W)$, associe $f^{\sharp} \in \Gamma_{0}(W)$ telle que : $\quad f^{\sharp}=f^{*}(-\cdot)$ où $f^{*}$ est la conjuguée de $f$.

On vérifie facilement que, si $f, g \in \Gamma_{0}(W)$, alors :

$$
\left(\frac{f+g}{2}\right)^{\sharp} \leq \frac{f^{\sharp}+g^{\sharp}}{2} \text {. }
$$

On considère l'algorithme suivant :

A partir de $f_{1}$ et $g_{1}$ donnés appartenant à $\Gamma_{0}(E)$, on définit deux suites d'éléments de $\Gamma_{0}(E)$, à savoir $\left(f_{p}\right)$ et $\left(g_{p}\right)$ tels que :

On montre que :

$$
\forall p \in \mathbb{N}^{*}, f_{p+1}=\frac{f_{p}+g_{p}}{2}, \quad g_{p+1}=\left(\frac{f_{p}^{\sharp}+g_{p}^{\sharp}}{2}\right)^{\sharp}
$$

Proposition 7.1. si dom $f_{2}=$ dom $g_{2}$, alors, les suites $\left(f_{p}\right)_{p \in \mathbb{N}}$ et $\left(g_{p}\right)_{p \in \mathbb{N}}$ convergent vers la même limite et l'on $a$ :

$$
\lim _{p \rightarrow+\infty} g_{p}=\sup _{p \in \mathbb{N}} g_{p}=g_{\infty}=f_{\infty}=\inf _{p \in \mathbb{N}} f_{p}=\lim _{p \rightarrow+\infty} f_{p}
$$

D'autre part, posons :

$$
\forall f, g \in \Gamma_{0}(E), \frac{f+g}{2}=f \alpha_{1} g\left(\operatorname{resp} .\left(\frac{f^{\sharp}+g^{\sharp}}{2}\right)^{\sharp}=f \alpha_{1}^{\sharp} g\right),
$$

et

Alors :

$$
\alpha_{p+1}=\frac{\alpha_{p}+\alpha_{p}^{\sharp}}{2}
$$

$$
\forall f, g \in \Gamma_{0}(E), \forall p \in \mathbb{N}^{*}, f_{p+1}=\frac{f_{1} \alpha_{p} g_{1}}{2}, g_{p+1}=\frac{f_{1} \alpha_{p}^{\sharp} g_{1}}{2}
$$

Ainsi, la suite $\left(\alpha_{p}\right)_{p \in \mathbb{N}}$ tend vers une limite $\alpha_{\infty}$ lorsque $p$ tend vers l'infini et :

$$
f_{\infty}=g_{\infty}=f_{1} \alpha_{\infty} g_{1}=f_{1} \alpha_{\infty}^{\sharp} g_{1} .
$$

Proposition 7.2. Soit $(E, p)$ (resp. $(F, q))$ un espace vectoriel semi-normé et $E^{*}\left(r e s p . F^{*}\right)$ son dual topologique. Notons $\langle\cdot, \cdot\rangle_{\otimes}$ le crochet de dualité entre $E^{*} \otimes F^{*}$ et $E \otimes F$.

Soit $f_{0} \in \overline{\mathbb{R}}_{+}^{E}$ et $g_{0} \in \overline{\mathbb{R}}_{+}^{F}$ telles que $f_{0}^{\sharp} \in \overline{\mathbb{R}}_{+}^{E^{*}}$ et $g_{0}^{\sharp} \in \overline{\mathbb{R}}_{+}^{F^{*}}$.

Posons :

$$
f_{1}=f_{0} \pi g_{0}, g_{1}=f_{0} \varepsilon g_{0}=\left(f_{0}^{\sharp} \pi g_{0}^{\sharp}\right)_{\otimes}^{\sharp},
$$


et

$$
\forall p \in \mathbb{N}^{*}, f_{p+1}=\frac{f_{p}+g_{p}}{2}, g_{p+1}=\left(\frac{f_{p}^{\sharp}+g_{p}^{\sharp}}{2}\right)_{\otimes}^{\sharp} .
$$

Si, relativement à la dualité définie par le crochet $\langle\cdot, \cdot\rangle_{\otimes}, f_{1} \in \Gamma_{0}(E \otimes F)$,

alors, les suites $\left(f_{p}\right)_{p \in \mathbb{N}}$ et $\left(g_{p}\right)_{p \in \mathbb{N}}$ convergent, lorsque $p$ tend vers l'infini, vers une même limite que nous noterons $f_{0} \rho g_{0}$ et l'on aura alors :

$$
f_{0} \rho g_{0}=\left(\left(f_{0} \pi g_{0}\right) \alpha_{\infty}\left(f_{0} \epsilon g_{0}\right)\right)=f_{0} \rho^{\sharp} g_{0} .
$$

\section{Propriétés topologiques de $f \pi g$ et $f \varepsilon g$}

\section{Remarques préliminaires.}

(1) La définition de $f \pi g$ est algébrique.

(2) La définition de $f \varepsilon g$ dépend des semi- normes $p$ de $E$ et $q$ de $F$.

(3) $E \otimes F$ et $E^{*} \otimes F^{*}$ sont mis en dualité par le crochet de dualité $\left.<\cdot, \cdot\right\rangle_{\otimes}$. Notons $\sigma\left(E \otimes F, E^{*} \otimes F^{*}\right)$ la topologie faible sur $(E \otimes F)$ associée au crochet $\langle\cdot, \cdot\rangle_{\otimes}$ et $(E \otimes F)_{\sigma}$ l'espace $(E \otimes F)$ muni de la topologie $\sigma\left(E \otimes F, E^{*} \otimes F^{*}\right)$.

Il résulte, de sa définition, que $f \varepsilon g$ est s.c.i. $\operatorname{sur}(E \otimes F)_{\sigma}$.

Il en va différemment de $f \pi g$.

On peut montrer que, en général,

$$
(f \text { s.c.i. } \operatorname{sur}(E, p) \text { et } g \text { s.c.i. } \operatorname{sur}(F, q)) \not\left(f \pi g \text { s.c.i. } \operatorname{sur}(E \otimes F)_{\sigma}\right) \text {, }
$$

et même que, en général,

$$
(f \text { s.c.i. } \operatorname{sur}(E, p) \text { et } g \text { inf-compacte } \operatorname{sur}(F, q)) \not\left(f \pi g \text { s.c.i. } \operatorname{sur}(E \otimes F)_{\sigma}\right) \text {. }
$$

Par contre:

(a) $\mathbf{s i}(E \otimes F)_{\sigma}$ est quasi-complet,

(b) si $f$ est inf-compacte $\operatorname{sur}(E, p)$ et $\mathbf{s i} g$ est inf-compacte $\operatorname{sur}(F, q)$ alors $\left(f \pi g\right.$ est s.c.i. $\operatorname{sur}(E \otimes F)_{\sigma}$ ou encore :

(c) si $A$ est une partie de $E$ faiblement compacte dans $(E, p)$, si $B$ est une partie de $F$ faiblement compacte dans $(F, q)$ alors $A \otimes B$ est compacte dans $(E \otimes F)_{\sigma}$

(d) si , de plus, $(E \otimes F)_{\sigma}$ est quasi complet, alors $\overline{c o}(A \otimes B)$ est compacte dans $(E \otimes F)_{\sigma}$. (On rappelle qu'un espace vectoriel topologique est quasi-complet si toute partie bornée fermée de cet espace est complète).

(4) On sait que :

$$
\left(f \varepsilon g=\left(f^{\sharp} \pi g^{\sharp}\right)_{\otimes}^{\sharp}\right) \Longleftrightarrow\left(\varepsilon=(\pi)_{\otimes}^{\sharp}\right)
$$

D'autre part :

$$
\left(f^{\sharp} \varepsilon g^{\sharp}\right)_{\otimes}^{\sharp}=\left(\left(f^{\sharp \sharp} \pi g^{\sharp \sharp}\right)_{\otimes}^{\sharp}\right)_{\otimes}^{\sharp},
$$

donc, si $f \in \Gamma_{0}(E)$ et $g \in \Gamma_{0}(F)$, alors :

$$
\left(f^{\sharp} \varepsilon g^{\sharp}\right)_{\otimes}^{\sharp}=(f \pi g)_{\otimes}^{\sharp \sharp} .
$$

Et si $f \pi g$ est s.c.i. sur $(E \otimes F)_{\sigma}$, alors :

$$
f \pi g=f\left((\varepsilon)_{\otimes}^{\sharp}\right) g .
$$


(5) A. Grothendieck dans [6] a montré que : Quelle que soit la semi-norme p sur l'espace vectoriel E et quelle que soit la semi-norme q sur l'espace vectoriel $F$ on $a$ :

$$
p \varepsilon q=p\left((\pi)_{\otimes}^{\sharp}\right) q \text { et } p \pi q=p\left((\varepsilon)_{\otimes}^{\sharp}\right) q,
$$

et quels que soient les espaces vectoriels $E$ et $F$, quel que soit $z \in E \otimes F$, quel que soit $\gamma$ tel que $\varepsilon \leq \gamma \leq \pi$ :

$$
(p \gamma q)(z)=\operatorname{Inf}\left\{(p \gamma q)(u) ; u \in M \otimes N, M \in \mathcal{V}_{f}(E), N \in \mathcal{V}_{f}(F)\right\}
$$

où $\left.\mathcal{V}_{f}(E)\right) \quad\left(\right.$ resp. $\left.\mathcal{V}_{f}(F)\right)$ est l'ensemble des sous-espaces vectoriels de $E$ (resp. $F$ ) de dimensions finies.

Il apparaît ainsi que les propriétés de $p \gamma q$ sur des espaces vectoriels de dimensions quelconques se déduisent de ses propriétés sur les espaces de dimensions finies.

Définition 8.1. Suivant A. Grothendieck, nous associerons à tout produit tensoriel $\gamma$ de fonctionnelles définies sur les espaces de dimensions quelconques le produit tensoriel $\widetilde{\gamma}$ tel que:

Quels que soient les espaces de Banach $(E, p)$ et $(F, q)$, quelles que soient les fonctionnelles $f \in \overline{\mathbb{R}}_{+}^{E}$ et $g \in \overline{\mathbb{R}}_{+}^{F}$ telles que $f^{\sharp} \in \overline{\mathbb{R}}_{+}^{E^{*}}$ et $g^{\sharp} \in \overline{\mathbb{R}}_{+}^{F^{*}}$, quel que soit $z \in E \otimes F$,

$$
\left.(f \widetilde{\gamma} g)(z)=\operatorname{Inf}\left(f_{M} \gamma g_{N}\right)(u) ; u \in M \otimes N, M \in \mathcal{V}_{f}(E), N \in \mathcal{V}_{f}(F)\right)
$$

où $\mathcal{V}_{f}(E) \quad\left(\right.$ resp. $\left.\mathcal{V}_{f}(F)\right)$ est l'ensemble des sous-espaces vectoriels de $E$ (resp.F) de dimensions finies et $f_{M}$ (resp. $g_{N}$ ) est la restriction de $f$ (resp.g) à $M$ (resp. $\left.N\right)$.

On dira que $\widetilde{\gamma}$ est de type fini si $\widetilde{\gamma}=\gamma$.

On sait que : $\widetilde{\varepsilon} \leq \tilde{\pi}$.

On dira que $\gamma$ est raisonnable si : $\widetilde{\varepsilon} \leq \widetilde{\gamma} \leq \widetilde{\pi}$.

$\rho$ étant le produit tensoriel défini dans la proposition 7.2 , on a :

Proposition 8.1. $\widetilde{\varepsilon} \leq \widetilde{\rho} \leq \widetilde{\pi}$.

\section{REFERENCES}

[1] M. Atteia, An optimal tensor convex control problem, International Journal of Pure and Applied Mathematics, Vol. 9, N1, 2003, 125-133

[2] M. Atteia et M. Raissouli, Self dual operators on convex functionals. Geometric mean and square root of convex functionals, Journal of convex analysis, Volume 8, 2001, n1

[3] J-P. Dedieu, Etude d'un point de vue projectif des fonctionnelles convexes. Produits tensoriels des fonctionnelles convexes, Université Paul Sabatier, juin 1975.

[4] A. El Qortobi, Contribution la dualité pour les fonctionnelles quasi-convexes, Université Paul Sabatier, janvier 1980.

[5] A. Grothendieck, Produits tensoriels topologiques et espaces nucléaires, AMS, $1955 \rightarrow 1966$ (4º́dition).

[6] A. Grothendieck, Résumé de la théorie métrique des produits tensoriels topologiques, Sao-Paulo (Brésil),1956.

[7] G. Köthe, Topological vector spaces II , Springer-Verlag,Band 159, 1969.

[8] Raymond A. Ryan, Introduction to tensor products of Banach spaces, Springer-Verlag, 2002.

[9] R. Schatten, A theory of cross spaces, Princeton, 1950.

C'est John Von Neumann qui est à l'origine de tous les travaux sur les produits tensoriels topologiques. 